\title{
L'UdPPC et la SFP célèbrent ensemble le cinquantenaire de la découverte du laser
}
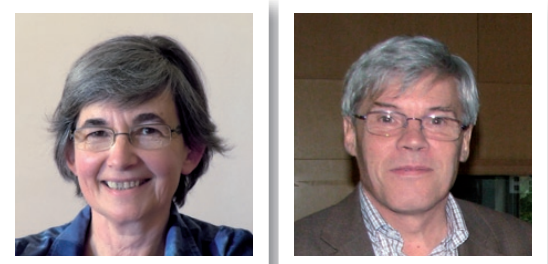

Le Laser a 50 ans. Suite à la première démonstration d'un effet laser par Theodore Maiman en mai 1960, et en dépit de prédictions hâtives (« le laser, une solution à la recherche de son problème »), les lasers ont connu un développement exponentiel : les sources se sont multipliées dans tous les domaines de longueur d'onde, de l'infrarouge lointain aux rayons $X$ durs, établissant des records de monochromaticité, cohérence spatiale et pureté spectrale, de durée ultra-courte d'impulsions lumineuses, de puissance, d'énergie... Leurs applications ont été multiples en recherche fondamentale (physique, chimie, etc.), dans l'industrie, dans les télécommunications, en métrologie, en biologie et médecine... À cause de ces nombreuses applications, le laser est devenu un élément de notre vie quotidienne. En tant que source lumineuse quasi idéale, il a aussi révolutionné l'enseignement des sciences physiques et de l'optique.

Pour célébrer les 50 ans du laser, présenter les récentes avancées et décrire son impact dans les sciences physiques et ses nombreuses applications dans les autres disciplines, la Société française de physique et l'Union des professeurs de physique et de chimie ont uni leurs forces pour publier un numéro spécial commun de leurs revues respectives, Reflets de la physique et Le Bulletin de I'Union des Professeurs de physique et de chimie. La lecture de son sommaire montre la diversité des thèmes abordés.

Il faut souligner que la présente publication s'inscrit dans un ensemble d'initiatives communes prises par nos deux sociétés depuis de nombreuses années. En juin 2005, à l'occasion de l'Année mondiale de la physique, la SFP et l'UdPPC avaient publié en commun un numéro hors série du Bup et du Bulletin de la Société française de physique, ancêtre des Reflets de la physique. Ce numéro mettait en relief quelques-uns des champs d'activité des physiciens d'aujourd'hui et leurs liens avec les disciplines les plus diverses. Édouard Brézin, alors vice-président de la SFP, soulignait dans la préface que cette publication témoignait de "la vitalité de la physique contemporaine, la diversité de ses objets d'étude, la richesse de ses contacts avec d'autres disciplines ». Madeleine Sonneville, présidente de I'UdPPC, soulignait de son côté, "l'importance de tels documents pour la culture scientifique des professeurs ".

Si cette publication commune était une «première » dans le domaine éditorial, elle ne représentait pas la première action commune des deux associations. Les lecteurs assidus de nos bulletins se rappellent sûrement qu'en 1986 avait pris naissance une commission mixte UdPPC-SFP sur l'enseignement. Elle a poursuivi ses travaux pendant de longues années, mais fut surtout le lieu d'élaboration du projet des Olympiades de Physique France. Celui-ci vit le jour en septembre 1991, lors du congrès général de la SFP à Caen. Les deux sociétés, cofondatrices des Olympiades, en sont aussi les coorganisatrices, et les deux revues se font chaque année l'écho du concours. Au-delà de cet exemple, notre volonté de collaboration est constamment réaffirmée et elle trouve en particulier à s'illustrer par des échanges réguliers entre les associations, par des articles publiés en commun et aussi (trop souvent, malheureusement...) chaque fois que sont malmenées les conditions faites à l'enseignement des sciences au lycée.

Nos remerciements s'adressent à tous les auteurs qui ont accepté de contribuer, et aux équipes de rédaction qui se sont coordonnées pour mener à bien ce projet ambitieux, afin d'aboutir à ce beau numéro que nous sommes heureux de vous présenter et qui devrait faire référence.

Micheline Izbicki

Présidente de l'UdPPC
Martial Ducloy

Président de la SFP 